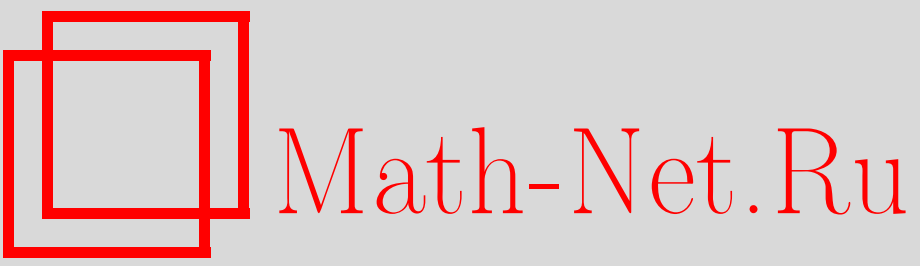

Ю. С. Колесов, А. А. Кочурин, Высокомодовый параметрический резонанс в задаче о повышении устойчивости за счет вибраций упругих систем, УМН, 1999, том 54, выпуск 5, 161-162

DOI: https://doi.org/10.4213/rm210

Использование Общероссийского математического портала Math-Net.Ru подразумевает, что вы прочитали и согласны с пользовательским соглашением

http: //www . mathnet.ru/rus/agreement

Параметры загрузки:

IP : 35.173 .219 .149

26 апреля 2023 г., 13:27:22 


\title{
ВЫСОКОМОДОВЫЙ ПАРАМЕТРИЧЕСКИЙ РЕЗОНАНС \\ В ЗАДАЧЕ О ПОВЫШЕНИИ УСТОЙЧИВОСТИ ЗА СЧЕТ ВИБРАЦИЙ УПРУГИХ СИСТЕМ
}

\author{
Ю. С. КолеСов, А. А. Кочурин
}

1. Данный вопрос впервые был поставлен в статье [1], а способ его решения - исследование устойчивости соответствующих дифференциальных уравнений второго порядка, получающихся при проектировании на моды - собственные функции - с небольшими номерами. Ниже приводятся результаты исчерпывающего анализа, когда к рассмотрению привлекаются моды с произвольными номерами.

Для простоты изложения ограничимся краевой задачей

$$
\begin{gathered}
\frac{\partial^{2} u}{\partial t^{2}}+\alpha \frac{\partial u}{\partial t}+\left(-\gamma^{2}+\frac{\beta}{\varepsilon} \sin \frac{t}{\varepsilon}\right) u=\frac{\partial^{2} u}{\partial x^{2}} \\
\left.u\right|_{x=0}=\left.u\right|_{x=\pi}=0
\end{gathered}
$$

где $\varepsilon$ - малый положительный параметр, коэффициент затухания $\alpha>0$, постоянная $\gamma>1$, параметр $\beta$ - характеристика вибрационного воздействия. Следует обратить внимание на то, что при $\beta=0$ на некотором числе младших мод имеем экспоненциальное возрастание решений. Ставится вопрос: можно ли за счет вибрационного воздействия добиться экспоненциального затухания всех решений краевой задачи (1), (2), начальные условия которых обладают стандартными условиями гладкости? В [1] ответ на этот вопрос считается положительным. Мы же покажем, что по тенденции он скорее отрицательный.

2. Привлекая метод Фурье, вместо краевой задачи $(1),(2)$ получаем счетное число обыкновенных дифференциальных уравнений

$$
\frac{d^{2} u}{d t^{2}}+\alpha \frac{d u}{d t}+\left(-\gamma^{2}+\frac{\beta}{\varepsilon} \sin \frac{t}{\varepsilon}\right) u+k^{2} u=0, \quad k=1,2, \ldots
$$

ТЕорема 1. Пусть параметр $\gamma$ таков, что

$$
\gamma^{2}<\frac{\beta^{2}}{2}+1
$$

Тогда найдутся такие положительнье постояннье $\varepsilon_{0} u m, M$, где $m<M$, что при $0<\varepsilon \leqslant \varepsilon_{0}$ әкспоненциально затухают решения каждого из уравнений (3) с номерами $k \leqslant m / \varepsilon u k \geqslant M / \varepsilon$.

3. Перейдем к случаю, когда

$$
\frac{m}{\varepsilon}<k<\frac{M}{\varepsilon} .
$$

Оказывается, что при ограничении (5) основной интерес представляют моды с номерами

$$
k=\frac{1}{2 \varepsilon}+\delta,
$$

где зависящая от $\varepsilon$ постоянная $\delta$ ограничена при $\varepsilon \rightarrow 0$. Дело в том, что на таких модах в уравнении (3) возможна параметрическая неустойчивость.

Работа выполнена при финансовой поддержке Российского фонда фундаментальных исследований (грант № 99-01-00549). 
TeOpema 2. Пусть

$$
\frac{\beta^{2}}{4}>\alpha^{2}+\frac{1}{4}
$$

Тогда найдется такое $\varepsilon_{0}$, что при $0<\varepsilon \leqslant \varepsilon_{0}$ некоторое конечное число уравнений (3), номера которых подчинены неравенствам (5), имеет әкспоненциально растущие решения с не зависящим от в показателем әкспоненты.

TeOpema 3. Пусть

$$
|\beta|<2 \alpha
$$

Тогда найдется такое $\varepsilon_{0}>0$, что при $0<\varepsilon \leqslant \varepsilon_{0}$ әкспоненциально затухают решения каждого из уравнений (3), номера которых подчинены оченкам (5).

TEOPEMA 4. Пусть

$$
\alpha^{2}<\frac{\beta^{2}}{4}<\alpha^{2}+\frac{1}{4}
$$

Тогда при стремлении є к нулю чередуются свойства устойчивости и неустойчивости некоторых уравнений (3), номера которых подчинены неравенствам (5).

4. Достаточно просты доказательства всех сформулированных предложений. Их идейная суть такова. После перехода к медленному времени $\varepsilon t$ при небольших номерах $k$ доказательство теоремы 1 сводится к применению алгоритма из [2]. Он же используется при больших номерах $k, o$ которых идет речь в теореме 1 . Наконец, до номеров порядка $1 / \varepsilon$ утверждение теоремы "дотягивается" путем использования известной теоремы Ляпунова [3].

Доказательства остальных утверждений также опираются на описанный в [2] алгоритм.

Теперь об общем физическом выводе. Только при одновременном выполнении неравенств (4) и (8) реализуется классический феномен повышения устойчивости за счет вибраций. Следует также еще раз подчеркнуть, что в силу неравенства (8) в случае упругих систем он принципиально другой: повышение устойчивости не есть прямолинейное следствие увеличения амплитуды вибрационного воздействия.

\section{СПИСОК ЛИТЕРАТУРЫ}

[1] Челомей В. Н. // Докл. АН СССР. 1956. Т. 110. № 3. С. 345-347. [2] Мищенко Е. Ф., Колесов Ю. С., Колесов А. Ю., РОзов Н. Х. Периодические движения и бифуркационные процессы в сингулярно возмущенных системах. М.: Физматлит, 1995. [3] Якубович В. А., Старжинский В. М. Линейные дифференциальные уравнения с периодическими коэффициентами и их применения. М.: Наука, 1972. 